\title{
Towards Support Processes for Web Projects
}

\author{
Pablo Becker and Luis Olsina \\ GIDIS_Web, Engineering School, UNLPam \\ Calle 9 y 110, (6360) Gral. Pico, La Pampa, Argentina \\ \{beckerp, olsinal\}@ing.unlpam. edu.ar
}

\begin{abstract}
Measurement, evaluation and analysis are support processes to main engineering processes. In this work we present an integrated strategy whose rationale is supported by a well-defined measurement and evaluation process, a conceptual framework that relies on an ontological base, and quality evaluation methods. Particularly, we discuss some process views for specifying project context, nonfunctional requirements, measurement, evaluation and analysis. Finally, the benefits of having well-established measurement and evaluation processes as quality drivers for web projects are highlighted.
\end{abstract}

Keywords: Process, Measurement, Evaluation, Quality, C-INCAMI, WebQEM.

\section{Introduction}

Quality, scope, time and cost are the main interdependent variables for managing software/web development projects. For each engineered project, and independently of the development lifecycle adopted, levels of quality for its entities and attributes should be agreed, specified, measured and evaluated for analyzing and improving them. To assure repeatability and consistency of results, it is necessary to have welldefined measurement and evaluation (M\&E) support processes with clearly established activities, sequences, inputs and outputs, roles, etc.

Given the inherent complexity of the process domain, a process can be modeled taking into account different views [4], namely: i) Functional, which includes the activities' structure, inputs and outputs, etc.; ii) Informational that includes the structure and interrelationships among artifacts produced or consumed by the activities; iii) Behavioral, which models the dynamic view of processes; and, iv) Organizational that deals with agents, roles and responsibilities. For specifying all the views, different modeling languages can be used. Each language has its own strengths and weaknesses that can make it more suitable for modeling certain views than others. In this work we focus on functional and behavioral perspectives of the M\&E process, using UML activity diagrams and the SPEM Profile [17]. Activity diagram is a well-known standard widespread both in academic and industry, easy to understand, and also fits to our modeling needs for both views. We are planning to cover the integration of views using the SPEM approach.

Besides establishing a set of activities and procedures for specifying, collecting, storing, and using metrics (for measurement) and indicators (for evaluation), we argue that more robust analysis and decision-making process can be achieved if the following 
three aspects of an evaluation strategy are considered at once [15]: i) a $M \& E$ process; ii) a conceptual framework that relies on an ontological base; and, iii) evaluation methods and tools instantiated from both the framework and process.

Firstly, to assure repeatability and reproducibility for M\&E activities and then consistent results, it is necessary to provide a well-defined process (model), which prescribes or informs a set of activities, their inputs and outputs, roles, interdependencies, and so forth. Secondly, a well-established conceptual framework should be built upon a robust base, which explicitly and formally specifies the main agreed concepts, properties, relationships, and constraints for the M\&E domain. Lastly, web quality evaluation methods and tools appropriately instantiated from both the process descriptions and framework components may be derived.

In previous works, we have built, based on a metrics and indicators ontology [13], a framework called C-INCAMI (Contextual-Information Need, Concept model, Attribute, Metric and Indicator) [15], and the WebQEM method (Web Quality Evaluation Methodology) [14] and its associated tool. This methodology was used in different case studies $[14,16]$. Nevertheless, the M\&E process was not explicitly and formally specified as we currently do it in the present work.

The modeling of these processes contributes to: i) facilitate the understanding and communication among stakeholders; ii) integrate and formalize different activities that are interrelated as nonfunctional requirements specification, context specification, M\&E design, analysis and recommendation; iii) reuse the ontological base stemming from the C-INCAMI framework easing understandability and interoperability; iv) allow the instantiation of methods and tools; and v) foster the integration with other processes such as testing and quality assurance.

The rest of this paper is organized as follows. Section 2 presents related work on M\&E processes. Section 3 gives an overview of the C-INCAMI framework. Section 4 discusses the functional view and to lesser extent the behavioral view of these processes, illustrating them by examples. Details of the C-INCAMI tool are given in Section 5 and, finally, the main remarks and future directions are outlined.

\section{Related Work and Discussion}

There exists an ongoing SQuaRE (Software product Quality Requirements and Evaluation) project that proposes harmonizing many ISO standards related to quality models, M\&E processes, etc. The ISO 25000 [10] document provides guidelines for the use of the new series of standards; however, the documents aimed at specifying $M \& E$ processes are not issued yet. So the standards for software measurement process (ISO 15939 [9]), and the process for evaluators (ISO 14598-5 [8]) are still in force. In [9] two activities out of four are considered to be the core measurement process, namely: Plan the measurement process, and Perform the measurement process. In [8] five activities are specified, namely: Establishment of evaluation requirements, Specification of the evaluation, Design of the evaluation, Execution of the evaluation plan, and Conclusion of the evaluation. In fact, we can observe that there is so far no unique ISO standard that specifies in an integrated way the M\&E process and approach as a whole. Moreover, in [13] we have shown that very often there is some lack of consensus about the used terminology among the quoted ISO standards as well as some missing terms, mainly for the evaluation domain. 
The CMMI (Capability Maturity Model Integration) [3] de facto standard is also worthy to mention in that it provides support process areas such as Measurement and Analysis, among others. It aligns information needs and measurement objectives with a focus on goal-oriented measurement -following to some extent the GQM (Goal Question Metric) [1] approach and the [9] measurement process. It specifies (specific/generic) practices to achieve the given process area goals. But, a process model itself is not defined; rather represents practices (i.e. actions/activities) without explicitly establishing sequences, parallelisms, control points, etc. Some specific practices for Measurement and Analysis are for example: Establish measurement objectives, Specify measures, Collect measurement data, and Analyze measurement data. However, a clear distinction between measurement and evaluation processes is missing in addition to lacking a robust conceptual base.

On the other hand, for the metrics and indicators domain there are several proposals regarding conceptual frameworks $[6,7,11]$. Unlike $[7,11]$, our conceptual framework (C-INCAMI) has an ontological root [13]. This ontology considered sources as ISO standards, articles and books, following the WebQEM terminology as well. Our ontology has similarities to the one presented in [5] and then slightly refined by some coauthors in [6], but in [15] we have modeled some terms (e.g., elementary indicator, global indicator, etc.) and relationships (e.g., measurement and measure, metric and indicator, among others) that differ semantically with those proposed in [6]. Also we have added new concepts, such as context, context properties, etc., not considered in the quoted works.

Ultimately, the main contribution of our research with regard to related work is a $M \& E$ strategy whose rationale is supported by integrated, well-defined M\&E processes, which in turn rely on a M\&E conceptual framework backed up by an ontological base. Next, an overview of our conceptual framework is given.

\section{C-INCAMI Components: An Overview}

The C-INCAMI framework provides a domain (ontological) model defining all the concepts and relationships needed to design and implement M\&E processes. It is an approach in which the requirements specification, M\&E design, and analysis of results are designed to satisfy a specific information need in a given context. In CINCAMI, concepts and relationships are meant to be used along the activities of M\&E processes. This way, a common understanding of data and metadata is shared among the organization's projects lending to more consistent analysis and results across projects.

Taking into account the main activities of the process (which are discussed in Section 4), the framework is structured in six components, namely: i) $M \& E$ project definition, ii) Nonfunctional requirements specification, iii) Context specification, iv) Measurement design and implementation, v) Evaluation design and implementation, and vi) Analysis and recommendation specification. For space reasons, just the components shown in Fig. 1 are presented below -for more details, see [15]. 


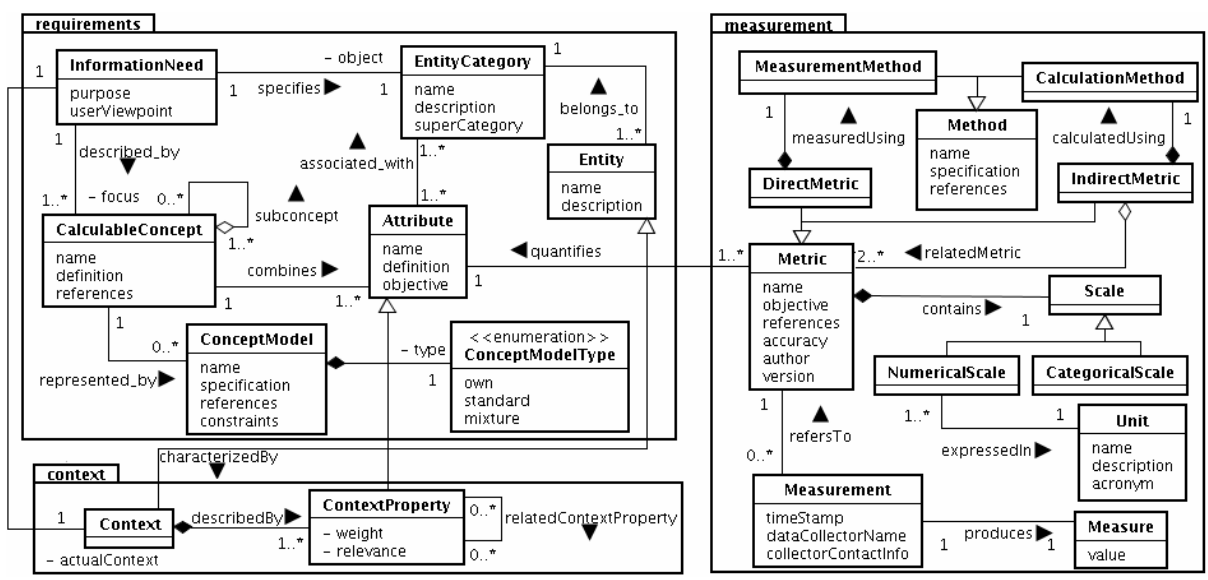

Fig. 1. Some concepts and relationships for the Nonfunctional requirements specification, Context specification, and Measurement design and implementation components

Nonfunctional requirements specification component (requirements package in Fig. 1) allows specifying the Information Need of a M\&E project. The information need identifies the purpose and the user viewpoint; in turn, it focuses on a Calculable Concept and specifies the Entity Category to evaluate -e.g. a resource, process, product, etc. A calculable concept can be defined as an abstract relationship between attributes of an entity and a given information need. For instance, external quality, quality in use, etc., are instances of a calculable concept. This can be represented by a Concept Model such as ISO software quality models. The leaves of an instantiated model (e.g. a requirement tree) are Attributes associated with an Entity.

In the Context specification component one concept is Context, which represents the relevant state of the situation of the entity to be assessed with regard to the information need. We consider Context as a special kind of Entity in which related relevant entities are involved. Consequently, the context can be quantified through its related entities that may be resources -as a network infrastructure, a working team, life-cycle processes-, the organization or the project itself, among others. To describe the context, attributes of the relevant entities are used -which are also Attributes called Context Properties. All context properties' metadata may be stored in an organizational repository so for each M\&E project the particular metadata and its values are stored as well.

The Measurement design and implementation component allows specifying the metrics that quantify attributes. To design a Metric the Measurement and Calculation Method and the Scale should be defined. Whereas a measurement method is applied to a Direct Metric, a calculation method is applied to an Indirect Metric. A Measurement produces a Measure as shown in Fig. 1.

\section{Modeling the Process for M\&E}

Process modeling is not a simple job. At the beginning of process modeling usually engineers think about what a process must do rather than how activities descriptions 
should be performed. So we can say that a process prescribes (or informs) a set of phases and activities, inputs and outputs, among other concerns, in order to foster repeatability and reproducibility. Taking into account the C-INCAMI framework and to some extent the ISO standards for M\&E, our integrated process proposal embraces the following main (core) activities: i) Define Non-Functional Requirements; ii) Design the Measurement; iii) Design the Evaluation; iv) Implement the Measurement; v) Implement the Evaluation; and vi) Analyze and Recommend.

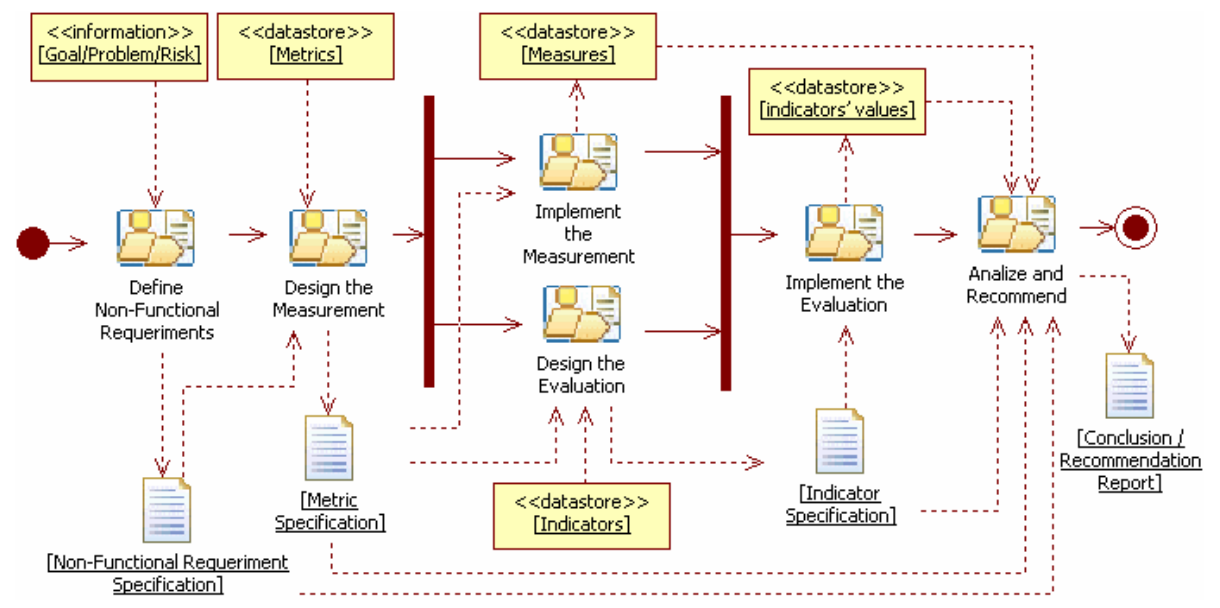

Fig. 2. Overview of the Measurement and Evaluation Process

These activities as well as sequences, parallelisms, inputs and outputs are shown in Fig. 2 using a terminological correspondence between the conceptual framework and process. The $<<$ datastore $>>$ stereotype represents repositories, e.g., the Metrics repository stores the metadata for the designed metrics.

The proposed $M \& E$ process (initially introduced in [2]) follows a goal-oriented approach. First, the Define Non-Functional Requirements activity has a specific goal or problem as input and a nonfunctional specification document as output. Then, the Design the Measurement activity allows identifying the metrics from the Metrics repository to quantify attributes: the output is a metric specification document. Once the measurement was designed, the evaluation design and the measurement implementation activities can be performed. The Design the Evaluation activity allows identifying indicators to be used. The Implement the Measurement activity uses the specified metrics to obtain the measures, which are stored in the Measures repository. Next, the Implement the Evaluation activity can be carried out. Finally, Analyze and Recommend activity has as inputs the values (i.e., data) of measures and indicators, the requirements specification document, and the associated metrics and indicators specifications (i.e., metadata).

In the next subsections, we illustrate the above six activities using excerpts of the case study developed in [16]. The case study dealt with the Cuspide.com shopping cart with the purpose of improving the external quality applying evaluation and WMR (Web Model Refactoring) in a systematic way. 


\subsection{Define Non-functional Requirements}

Once the requirement project has been created, Define Non-Functional Requirements is the first core activity to be performed as shown in Fig. 2. It consists of: Establish the Information Need, Specify the Context, and Select a Concept Model sub-activities as shown in Fig. 3 and described below.

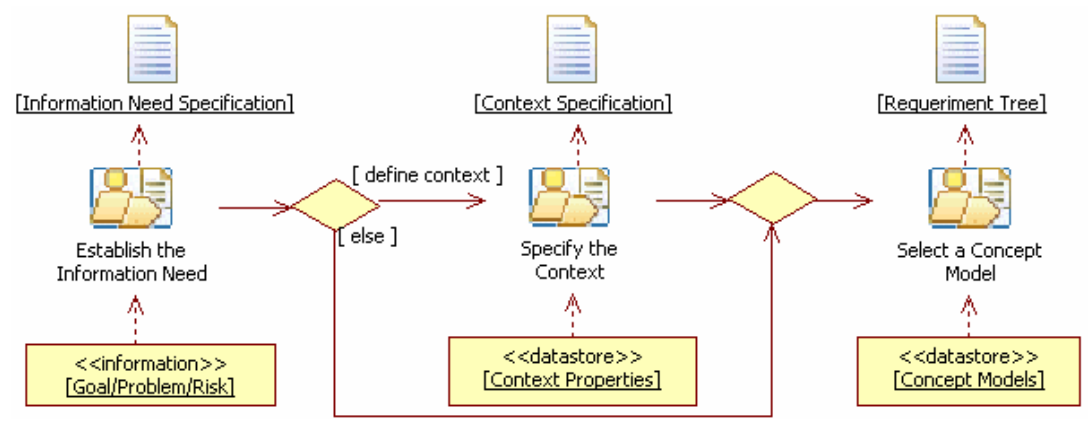

Fig. 3. Sub-activities for the Define Non-Functional Requirements activity

The Establish the Information Need activity is aimed at indicating the purpose, the user viewpoint, the focus, etc. of the M\&E project. Thus, we first Define the purpose (e.g. "improve" to our example); then Define the user viewpoint (e.g. "final user"), and Establish the object (e.g. "web application" as entity category). The next subactivity, Establish Entity, is aimed at identifying one or more concrete entities (e.g. "Cuspide.com shopping cart"), which belong to the selected entity category; however, the definition of the concrete entity can be deferred until measurement implementation. Note that it is possible to identify more than one entity for comparison purposes. Identify the focus is the final sub-activity in order to Establish the Information Need activity; e.g. the higher-level focus (CalculableConcept) is "external quality", while "usability" and "content quality" are the sub-concepts.

Once the information need specification document is yielded (Fig. 3), we optionally can Specify the Context as shown in Fig. 4. It involves to Select relevant Context Properties -from the organizational repository of context properties [12] - and, for each selected property the Quantify Context Property sub-activity must be performed based on the associated metric. In the end, we get as output a context specification document for a specific project. As example, the following relevant context properties are selected for the "refactoring project": "lifecycle type" whose value (based on the associated metric) is "Agile Methodology"; and "technique type" whose value is "WMR". Note that these context properties, i.e. the name, definition, value, etc. should be recorded with the M\&E project for further comparisons.

According to Fig. 3 Select a Concept Model is the last sub-activity to define nonfunctional requirements. It involves both Select a Model and Edit the Model subactivities. Concept models (e.g. the ISO 9126 internal and external quality models) are chosen from an organizational repository regarding the quality focus; in our example the external quality focus was considered. If the selected model is not totally suitable, e.g. some sub-characteristics or attributes are missing, it is necessary to Edit 
the Model, adding or removing sub-concepts and/or attributes accordingly. Table 1 shows, in the first column, an edited quality model (a requirement tree) for our study. Ultimately, the resulting artifact from the Define Non-Functional Requirements activity is the nonfunctional requirement specification document, which contains all the above mentioned information.

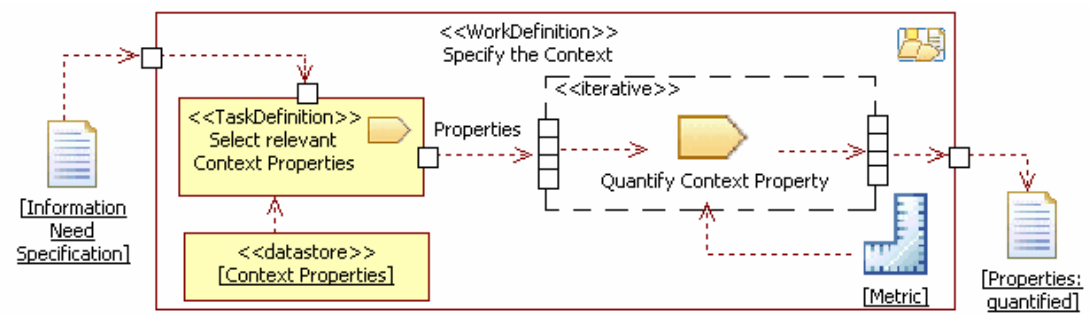

Fig. 4. Activities to Specify the Context

\subsection{Design the Measurement and Evaluation}

The Design the Measurement activity (recall Fig. 2) should be performed to identify suitable metrics regarding the nonfunctional requirement specification document. It consists of Establish Entity (still optional) and Assign one Metric to each Attribute activities (Fig. 5). It is important to remark that this activity does not mean designing each metric itself but selecting it from an organizational repository; however, if a needed metric is not there, then it should be designed, agreed by experts, and stored.

To Assign one Metric to each Attribute, first, we have to Identify a Metric that quantifies the attribute. The selected metric can be either direct or indirect. If the metric is indirect it is necessary to Identify related Metrics and Identify Attributes quantified by related Metrics. These two sub-activities allow identifying the extra attributes and metrics so that data collector may later gather data accordingly. Lately, if the measurement o calculation method of the metric can be automated the Select a Tool activity should be performed. Note in Fig. 5 that all these activities should be repeated for each attribute of the requirement tree as indicated by the $<<$ iterative $>>$ stereotype.

Regarding the case study, the attributes presented in table 1 are inputs to perform the Assign one Metric to each Attribute activity. For example, by performing the Identify a Metric sub-activity for the "Line item information completeness" attribute (defined as "the extent to which the line item information coverage is the sufficient amount of information to an intended user goal and task"), the direct metric named "Degree of completeness to the line item information" was assigned.

The selected metric scale specifies three categories considering an ordinal scale type, namely: (0) Incomplete, it has less information than category 1 or no information at all; (1) Partially complete, it only has title, price, quantity, and sometimes availability fields; and (2) Complete, it has title, author, price, quantity, added on date, and availability. The measurement method is objective and the data collection was made observationally. Because the selected metric is a direct metric, the Identify related Metrics and Identify Attributes quantified by related Metrics sub-activities are not necessary to be performed. As final output of these activities (Fig. 5) we get the metrics specification document. 


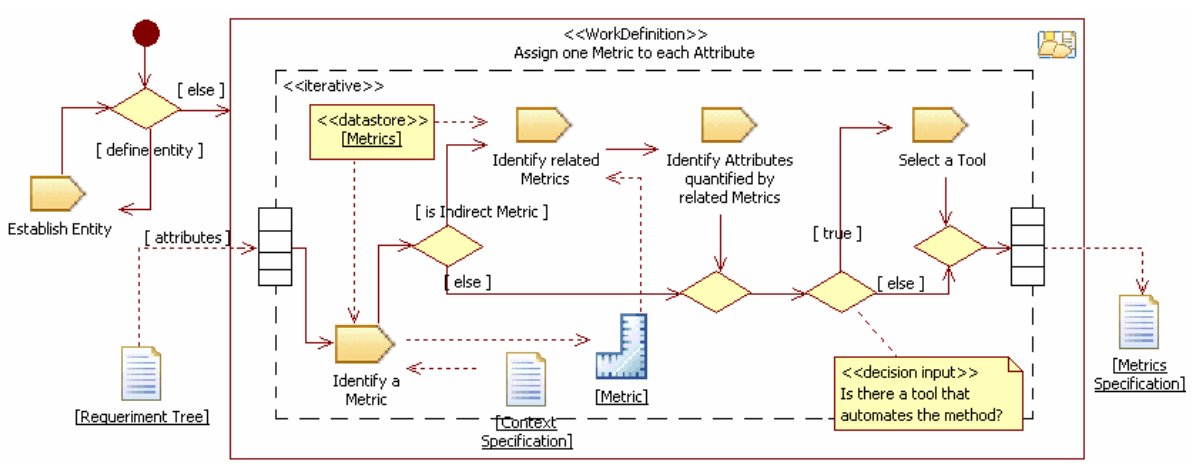

Fig. 5. The Design Measurement activity

On the other hand, the value of a particular metric does not necessarily represent the satisfaction level achieved by its associated attribute (that is an elementary nonfunctional requirement). Thus, we need to define a new mapping that will produce an elementary indicator value representing the achieved satisfaction level. Also we need to define partial and global indicators to evaluate the higher-level concepts of the requirement tree. Therefore we have to perform the Design the Evaluation activity as shown in Fig. 2. Particularly, Design the Evaluation entails two activities, namely: Identify Elementary Indicators and Identify Partial and Global Indicators.

The first activity allows specifying an indicator per each attribute (from the requirements tree) using the associated metric specification as input. For this, the involved sub-activities are: Establish the Elementary Model, Establish the Calculation Method (optional), and Identify the Scale. For example, for the "Line item information completeness" attribute, the elementary indicator named "Performance Level of the line item information completeness" was identified. For the elementary model, the following mapping from the metric specification is used: the measure value 0 maps to $0 \%, 1$ to $50 \%$, and 2 maps to $100 \%$. The decision criteria -in terms of acceptability ranges- were: unsatisfactory (red) for values within 0-40; marginal (yellow) for values within 40-70; and satisfactory (green) for values within 70-100.

Then, we perform the Identify Partial and Global Indicators activity for each concept in a similar way as shown above. The final output is an indicators specification document.

\subsection{Implement the Measurement and Evaluation}

The Implement the Measurement activity implies collecting all the measures for all attributes per each entity. In order to do this, the metrics specification document and the tools that automate the measurement and/or calculation methods are inputs. The involved activities are: Establish Entity and Measure Attributes. The activity Measure Attributes should be performed for each entity, recording the values, timestamps, etc. In our study, using the Degree of completeness to the line item information metric, the measurement produced the value 1 (see attribute 2.1.1.1 in table 1). All the measures are stored in the Measures datastore for further use. 
In a nutshell, for implementing the evaluation two activities are considered, viz. Calculate Elementary Indicators and Calculate Partial and Global Indicators. These activities must be performed for each entity. Looking at the design of the "Performance Level of the line item information completeness" indicator and the value 1 for the measure, which are inputs to the Calculate Elementary Indicators activity, an indicator value of $50 \%$ is yielded (see the third column of the table) as a consequence of applying the above elementary model. Once all the elementary indicators' values were obtained they serve as input to the Calculate Partial and Global Indicators activity. Then, the aggregation model is executed producing partial and global indicators' values. Some values are shown in the last column of table 1.

Table 1. Excerpt of the external quality model for a shopping cart [16]. Legend: EI stands for Elementary Indicator; P/GI stands for Partial/Global Indicator.

\begin{tabular}{|c|c|c|c|}
\hline Characteristics and Attributes (in italic) & Measure & EI value & P/GI value \\
\hline External Quality & & & $61.97 \%$ \\
\hline Usability & & & $60.88 \%$ \\
\hline & & & \\
\hline Content Quality & & & 63.05\% \\
\hline Content Suitability & & & $63.05 \%$ \\
\hline Basic Information Coverage & & & $50 \%$ \\
\hline Line item information completeness & 1 & $50 \%$ & \\
\hline Product description appropriateness & 1 & $50 \%$ & \\
\hline Coverage of other related Information & & & $76.89 \%$ \\
\hline Return policy information completeness & & $33 \%$ & \\
\hline
\end{tabular}

\subsection{Analyze and Recommend}

It is recognized that data, information, and knowledge are valuable assets for better organizational decision making. As such, data and metadata obtained from M\&E processes serve as inputs for analysis, conclusion and recommendation processes.

First, taking into account the business commitment, established information need, context, metrics and indicators specifications as well as the data properties with regard to the scale, the Design the Analysis activity (figure not shown) is performed. This activity involves deciding on the allowable mathematical and statistical methods and techniques for analysis regarding the scale type, datasets properties, etc., the suitable tools for the kinds of analysis at hand, the presentation and visualization mechanisms, and so forth.

Once the above design decisions have been made, the Implement the Analysis activity follows. Basically, it consists of implementing the designed procedures, running the planned tools and storing the results according to the established formats in order to produce the analysis report. Next by performing the Elaborate the Conclusion Report activity, this ends up drawing conclusions in the form of a conclusion report. However, decision makers very often need not only a conclusion report but also a recommendation report -which contains for instance strengths and weaknesses, course of actions to tackle the problems identified in the analysis, etc. 
For our case study, after looking at the conclusion report we were ready to start refactoring the navigation and presentation models to improve the Cuspide's shopping cart component. For example, one recommendation -stemming from the indicators' acceptability levels- was: changes should be planned in the "Basic Information Coverage" sub-characteristic (ranked 50\%), mainly in the "Line item information completeness" (2.1.1.1), and "Product description appropriateness" (2.1.1.2) attributes. A further course of action was performed by applying the Add Node Attribute, and Turn Information into Link refactoring models, which had impact in both attributes. As result of the improvement action, i.e. by executing the refactoring process, the enhanced shopping cart satisfied totally $(100 \%)$ these nonfunctional requirements -see details in [16].

\section{Technological Support for M\&E Projects}

With the aim of providing support to the third foundation mentioned in the Section 1 , i.e., evaluation methods and tools, the above discussed M\&E process and framework were instantiated in a web application called C-INCAMI Tool, which in turn gives technological support to the WebQEM methodology. The current tool version ${ }^{1}$ fully takes into account the specified M\&E process.

C-INCAMI Tool guides evaluators throughout the project definition, nonfunctional requirements specification, $M \& E$ design and implementation, and report generation activities showing results in a user-friendly way. The application presents to evaluators the activities that can be executed at any moment, enforcing the dependencies between them as defined by the process. Also it records the artifacts produced by the activities and inputs them to the required dependent activities. A screenshot of the application is shown in Fig. 6.

The architectural components of the tool are based on the conceptual components defined in the C-INCAMI framework (as shown in Section 3). These are responsible for implementing the business logic regarding the creation and manipulation of the Information Need, Concept Model, Metric, Indicator, among other concepts. Also, the tool allows creating and managing M\&E projects (called MEProject in CINCAMI framework [15]). Each MEProject contains a concrete Requirement Project; from this, one or more Measurement Projects can be created; and, in turn, for each measurement project one or more Evaluation Projects could be defined and created. So, for each M\&E project, subprojects can be managed accordingly. Each subproject is responsible for handling metadata and data to the corresponding conceptual component. This separation of concerns for each MEProject facilitates ultimately the traceability and consistency for ulterior intra- and inter-project analysis.

The web application has a multi-tier client-server architecture. For the persistence layer all repositories modeled in the process (recall $<<$ datastore $>>$ stereotype) were implemented using semantic web technologies, particularly, the Sesame RDF repository (www.openrdf.org/). In the presentation layer we have also used RIA (Rich Internet Application) technologies. Other employed technologies for the framework implementation were $\mathrm{Java}^{\mathrm{TM}}$. Ultimately, all documents are interoperable and usable among different analysis tools because they are specified in XML.

\footnotetext{
${ }^{1}$ Available at http://gidisw.ing.unlpam.edu.ar:8080/INCAMI-WS/
} 


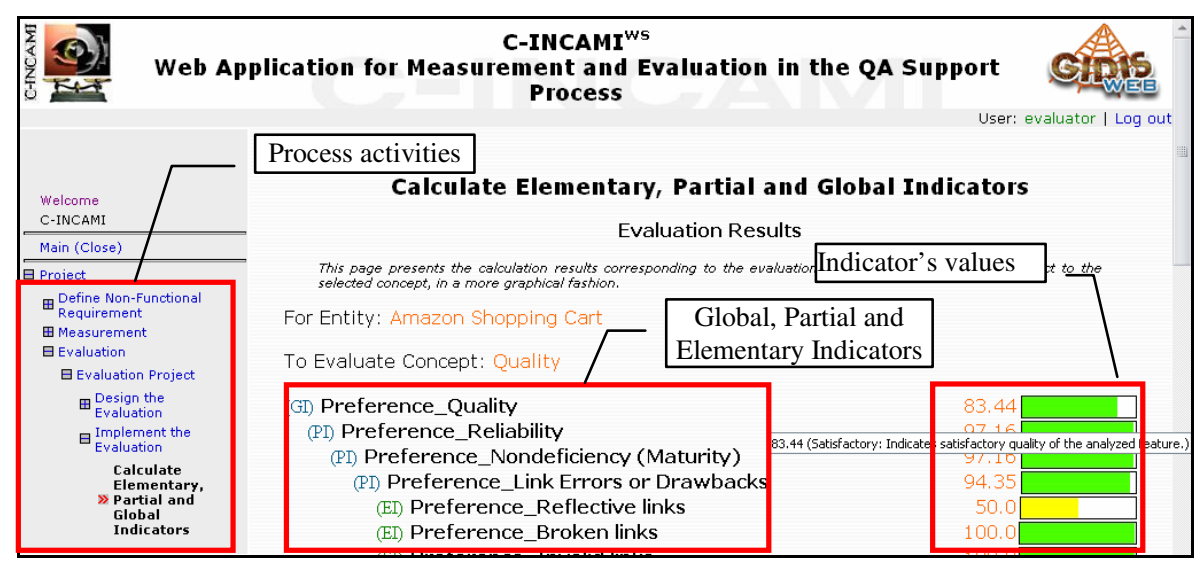

Fig. 6. C-INCAMI Tool screenshot showing results in the Evaluation activity

\section{Conclusion and Future Work}

Organizations relying only on engineering process areas and not paying enough attention to supporting process areas -as measurement, evaluation and analysis- are more prone to making less justifiable and repeatable projects and, therefore, more prone to fail. So having well-established strategies to cope with M\&E software/web projects and programs may be considered key quality drivers as well. This way, we have introduced our strategy based on three foundations viz. the M\&E process, the conceptual framework, and evaluation methods/tools. Particularly, we have addressed in this paper the specification of the M\&E process stressing in the Introduction Section the main contributions.

The underlying hypothesis of our proposal is that without well-established M\&E processes and without appropriate recorded metadata of concrete projects' information needs, context properties, attributes, metrics and indicators it is difficult to ensure data and datasets are repeatable and comparable among the organization's projects. As a consequence, analysis, comparisons and recommendations can be made in a less robust or even wrong way.

Finally, a humble contribution of this research with regard to ISO-related work is a $M \& E$ approach whose rationale is supported by interrelated and well-defined requirement, measurement, evaluation, analysis and recommendation processes. In addition, the discussed processes rely on a M\&E conceptual framework backed up by an ontological base. Also specific methods and tools can be instantiated from both the defined M\&E process and the C-INCAMI framework.

A future work is devoted to deal with modeling the remainder views -not just the functional and behavioral views as we have discussed here- and their integration to the C-INCAMI Tool.

Acknowledgements. This work and line of research is supported by the PAE-PICT 2188 project at UNLPam, from the Science and Technology Agency, Argentina. 


\section{References}

1. Basili, V., Rombach, H.D.: The TAME Project: Towards Improvement-Oriented Software Environments. IEEE Transactions on Software Engineering 14(6), 758-773 (1989)

2. Becker, P., Molina, H., Olsina, L.: Integrating Process and Measurement and Evaluation Framework. In: CIbSE 2009, Medellin, Colombia, pp. 261-266 (2009) (in Spanish)

3. Capability Maturity Model Integration, Version 1.2 CMMI for Development, CMU/SEI2006-TR-008 ESC-TR-2006-008, SEI Carnegie-Mellon University (2006)

4. Curtis, B., Kellner, M., Over, J.: Process Modelling. ACM Comm. 35(9), 75-90 (1992)

5. García, F., Ruiz, F., Bertoa, M.F., Calero, C., Genero, M., Olsina, L., Martín, M., Quer, C., Condori, N., Abrahao, S., Vallecillo, A., Piattini, M.: An ontology for software measurement. University of Castilla-La Mancha, Spain, TR. UCLM DIAB-04-02-2 (2004)

6. García, F., Bertoa, F., Calero, C., Vallecillo, A., Ruiz, F., Piattini, M., Genero, M.: Towards a consistent terminology for software measurement. Information \& Software Technology 48(8), 631-644 (2006)

7. Goethert, W., Fisher, M.: Deriving Enterprise-Based Measures Using the Balanced Scorecard and Goal-Driven Measurement Techniques. Software Engineering Measurement and Analysis Initiative, CMU/SEI-2003-TN-024 (2003)

8. ISO/IEC 14598-5. IT - Software product evaluation - Part 5: Process for evaluators (1998)

9. ISO/IEC 15939. Software Engineering - Software Measurement Process (2002)

10. ISO/IEC 25000. Software Engineering - Software product Quality Requirements and Evaluation (SQuaRE) - Guide to SQuaRE (2005)

11. Kitchenham, B.A., Hughes, R.T., Linkman, S.G.: Modeling Software Measurement Data. IEEE Transactions on Software Engineering 27(9), 788-804 (2001)

12. Molina, H., Olsina, L.: Assessing Web Applications Consistently: A Context Information Approach. In: IEEE CS, $8^{\text {th }}$ Int'l Congress on Web Engineering, NY, US, pp. 224-230 (2008)

13. Olsina, L., Martin, M.: Ontology for Software Metrics and Indicators. Journal of Web Engineering 2(4), 262-281 (2004)

14. Olsina, L., Rossi, G.: Measuring Web Application Quality with WebQEM. IEEE Multimedia 9(4), 20-29 (2002)

15. Olsina, L., Papa, F., Molina, H.: How to Measure and Evaluate Web Applications in a Consistent Way. In: Rossi, Pastor, Schwabe, Olsina (eds.) Springer HCIS book Web Engineering: Modeling and Implementing Web Applications, pp. 385-420 (2008)

16. Olsina, L., Rossi, G., Garrido, A., Distante, D., Canfora, G.: Web Applications Refactoring and Evaluation: A Quality-Oriented Improvement Approach. Journal of Web Engineering 7(4), 258-280 (2008)

17. SPEM. Software Process Engineering Metamodel Specification. Doc./02-11-14., Ver.1.0 (2002) 